\title{
Primary and acquired multidrug-resistant tuberculosis: Predictive factors for unfavorable treatment outcomes in Rio de Janeiro, 2000-2016
}

\author{
Marcela Bhering ${ }^{1}$ and Afrânio Kritski ${ }^{2}$
}

Suggested citation Bhering M, Kritski A. Primary and acquired multidrug-resistant tuberculosis: predictive factors for unfavorable treatment outcomes in Rio de Janeiro, 2000-2016. Rev Panam Salud Publica. 2020;44:e178. https://doi.org/10.26633/RPSP.2020.178

ABSTRACT Objective. To identify clinical and demographic factors associated with unfavorable treatment outcomes in patients with primary and acquired multidrug-resistant tuberculosis (MDR-TB) in Rio de Janeiro State.

Methods. Retrospective cohort study using data on 2269 MDR-TB cases in 2000-2016. Factors associated with unsuccessful, loss to follow-up, and death outcomes in patients with primary and acquired resistance were investigated with bivariate and multivariate regression.

Results. Primary resistance was $14.7 \%$ among MDR-TB cases. The unfavorable outcomes proportion was $30.3 \%$ in the primary resistance group and $46.7 \%$ in the acquired resistance group. There were significant differences in demographic and clinical characteristics between the two groups. Proportionally, the group with primary resistance had more cases among women (46.4\% vs. 33.5\% in the acquired resistance group), Caucasians (47.3\% and $34 \%$ ), and those with $\geq 8$ years of schooling (37.7\% and $27.4 \%)$. Extensively drug-resistant TB patients had 12.2-fold higher odds of unsuccessful outcome than MDR-TB patients, and comorbidities had 2-fold higher odds in the primary resistance group. Extensively drug-resistant TB had 5.43-fold higher odds in the acquired MDR-TB group. Bilateral disease and $<8$ years of schooling were associated with unsuccessful outcome in both groups. Being an inmate had 8-fold higher odds of loss to follow-up in the primary resistance group. Culture conversion by the sixth month was a protective factor for all outcomes.

Conclusions. Primary resistance cases of MDR-TB constitute a different transmission reservoir, which is related to other chronic diseases associated with higher acquisition of TB. The poor results observed in Rio de Janeiro State can contribute to increasing the transmission of primary MDR-TB, thus favoring drug resistance.

Keywords Tuberculosis; epidemiological monitoring; drug resistance; treatment outcome; Brazil.

The resistance of Mycobacterium tuberculosis (MTB) to drugs is a major threat and one of the challenges for disease elimination, given that it requires treatment with second-line regimen drugs, which are more expensive, more toxic, and less effective than the first-line regimen. Multidrug-resistant tuberculosis (MDR-TB) is defined as TB with resistance to at least rifampicin and isoniazid. In turn, extensively drug-resistant TB (XDR-TB) is defined as MDR-TB plus resistance to at least one quinolone and one of the three injectable drugs used in the treatment of TB (capreomycin, kanamycin, and amikacin) (1).

\footnotetext{
1 Sergio Arouca National School of Public Health, Oswaldo Cruz Foundation, Rio de Janeiro, Brazil $₫$ Marcela Bhering, marbhering@gmail.com
}

Although the main hypothesis for the increase in resistance cases had to do with treatment failures, some studies have shown that person-to-person transmission of MDR-TB is becoming more and more common, therefore resulting in a higher prevalence of primary MDR-TB (2).

The status of primary MDR-TB in Brazil is still poorly understood. The II National Survey on Resistance to Anti-TB Drugs, conducted in 2007-2008, indicated a primary and acquired resistance rate to isoniazid of $6.0 \%$ and $15.3 \%$, respectively. For rifampicin, these rates were $1.5 \%$ and $8.0 \%$, respectively.

\footnotetext{
${ }^{2}$ Federal University of Rio de Janeiro, Rio de Janeiro, Brazil
} 
MDR-TB rates, primary and acquired, were, respectively, $1.4 \%$ and $7.5 \%$ (3). There is, however, no study showing risk factors associated with unfavorable treatment outcomes for patients with primary MDR-TB.

That said, this study aims to identify the clinical and demographic factors associated with unfavorable treatment outcomes, specifically unsuccessful treatment, loss to follow-up (LTFU), and death of patients with primary and acquired MDR-TB in Rio de Janeiro State (RJ), Brazil's third most populous state (8.2\% of the country's population).

\section{MATERIALS AND METHODS}

\section{Research design and setting}

This retrospective cohort study used secondary data from the Special Tuberculosis Treatment Information System (SITETB) in RJ. In terms of incidence, Brazil ranks 12th worldwide in TB cases and 15th in TB-HIV coinfection, and is one of the 30 countries that the World Health Organization (WHO) considers priority for TB control (1). In 2017, Brazil reported 1041 cases of TB drug resistance, of which 713 (68.5\%) were MDR-TB (4). $\mathrm{RJ}$ is in southeast Brazil and has a population of approximately 17 million people. Its Human Development Index score is 0.761 , ranking fourth among Brazilian states (5). The average annual TB incidence rate in RJ was 63.3 and the mortality rate was 4.2 per 100000 population in 2018 (4).

\section{Tuberculosis surveillance system}

Brazil has had a disease notification system since 1975, whose quality has been improving since then. In 1993, the Notifiable Diseases Information System (SINAN) was created. The latter allows the notification, investigation, and monitoring of TB cases (6). In 2000, Brazil initiated epidemiological surveillance of MDR-TB, which, in turn, culminated in the implementation of an electronic information system, the SITETB (7). SITETB is used for the compulsory notification and follow-up of TB cases requiring special treatments for patients unable to use the standard TB regimen (2RHZE/4RH) (8). The records are made by health professionals and validated by professionals certified by the National Tuberculosis Program of the Ministry of Health (NTP-MoH). Demographic and clinical data, drug susceptibility testing (DST) results, adverse events, treatment regimens, and outcomes for each patient are registered (9). Whenever treatment with a special regimen is necessary, the case must be coded by SINAN as a regimen change, failure, or drug-resistant $\mathrm{TB}$, and needs to be notified in SITETB.

\section{Study sample}

In June 2019, the SITETB database contained information from a cohort of patients notified with MDR-TB in RJ from January 2000 to December 2016. Patients with the following attributes were excluded from the sample: those non-resident in RJ, undergoing treatment, with unknown outcomes, with a change in the diagnosis, or those with DST records not indicating resistance to, at least, rifampicin and isoniazid. Cases were divided into two groups according to the type of resistance: primary or acquired. According to NTP-MoH (8), the definitions are: Primary resistance is a new case of TB including (a) a patient never treated for TB; (b) a patient in treatment with a basic regimen, evolving to failure and subsequent diagnosis of drug-resistant TB; and (c) a patient in treatment with a basic regimen, who underwent culture and a drug sensitivity test at the beginning of treatment, with subsequent diagnosis of drug-resistant TB. Acquired resistance occurs if the patient reports previous TB treatments, except for the situations described in (b) and (c).

SITETB and SINAN do not have an interface. A manual linkage using Microsoft Excel was performed to check the consistency of the information about cases of primary resistance registered in SITETB. All cases with primary resistance were compared using patient name, their mother's name, and date of birth. If the patients had been previously notified and had not met criteria (b) and (c), the former were reclassified as acquired resistance.

\section{Treatment outcomes}

For the statistical analysis performed below, three treatment outcomes were operationalized as binary dependent variables: (i) unsuccessful; (ii) LTFU; and (iii) death. Treatment outcomes were classified according to the WHO definitions (10): Cured: the patient should have at least three negative cultures after the 12th month of treatment. Treatment completed: the patient completed the time stipulated for the treatment, with favorable clinical and radiological evolution, but without the follow-up cultures. LTFU: a patient whose treatment was discontinued for two consecutive months or more. Death: a patient who died for any reason during the treatment. Failure: two or more positive cultures out of the three recommended after the 12th month of treatment, or three consecutive positive cultures after the 12th month of treatment, at least 30 days apart. Failure may also be considered according to medical evaluation, and the decision to change treatment early due to clinical and radiological worsening. Unsuccessful: the sum of patients who had the outcome classified as death, LTFU, or failure.

\section{Independent variables}

The following independent variables were included on the right-hand side of the regression equations: sex, age under 40 years, less than eight years of schooling, race/skin color, HIV infection, diabetes, comorbidities (viral hepatitis, renal insufficiency, neoplasia, silicosis, transplant, mental disorder, prolonged use of corticosteroids, use of TNF-alpha inhibitors, seizure, and undefined others), illicit drug use, alcohol abuse, smoking, unemployment, inmate, drug resistance category (MDR-TB or XDR-TB), treatment regimen (standardized or individualized), the extent of disease (presence of chest cavity and/or bilateral disease), and six-month culture conversion. This last variable assigns the value 1 to patients who had at least two negative cultures until the sixth month after the start of treatment, value 0 otherwise. Only variables that had a maximum of $10 \%$ of missing values were selected.

\section{Statistical analysis}

According to the nature of each variable, number (frequency) and mean (standard deviation) were used to describe 
the characteristics of the patients with primary and acquired MDR-TB. The demographic and clinical characteristics were analyzed with descriptive statistics. Pearson's chi-squared test was employed for the comparison of categorical variables.

To verify the factors related to each outcome, a bivariate logistic regression was run. The crude odds ratio (OR) with $95 \%$ confidence intervals ( $95 \% \mathrm{CI}$ ) was calculated. Subsequently, the variables with $\leq 0.20$ significance were included in the multivariate logistic model. A backward method was performed using Hosmer-Lemeshow goodness of fit test for calibration and determining the adjusted odds ratio (ORa). Variables associated with outcomes with $\leq 0.10$ significance in the adjusted model were reported. Statistical analyses were performed using Stata software package version 13.1.

\section{Ethical considerations}

The study protocol was approved by the Research Ethics Committee of the Federal University of Rio de Janeiro (CAAE 10126919.2.0000.5257), which waived the need for written informed consent from participants as the study was based on secondary data and involved no more than minimal risk. Only one investigator (MB) had access to both identified and de-identified codes and prepared the anonymous database that was analyzed.

\section{RESULTS}

\section{Descriptive analysis}

Between 2000 and 2016, 2477 cases of MDR-TB were reported in SITETB RJ. In total 208 cases were excluded: 8 non-residents in RJ, 11 undergoing treatment, 3 unknown outcome, 11 diagnosis change, and 175 DST records not indicating resistance to, at least, rifampicin and isoniazid. Among the 2269 cases analyzed, 347 were recorded as cases of primary MDR-TB. Among these, 132 patients were reported in SINAN, and 13 were reclassified as acquired resistance. Of the total 2269 cases included in the sample, $334(14.7 \%)$ were primary resistance. The overall mean age was 39 years $( \pm 13.3), 1466(64.5 \%)$ were male, and 1281 (57.9\%) were living in Rio de Janeiro city, the capital of RJ.

Proportionally, the primary resistance group included more patients with the following characteristics than the acquired resistance group: women $(46.4 \%$ and $33.5 \%$, respectively); aged up to 24 years (22.2\% and $14 \%)$; Caucasian ( $47.3 \%$ and $34 \%)$; with eight or more years of schooling (37.7\% and $27.4 \%$ ); with diabetes $(13.2 \%$ and $9.0 \%)$; and with comorbidities $(14.3 \%$ and $11.6 \%$ ) (Table 1).

In addition, unemployment was more frequent in the acquired resistance group than in the primary resistance group (17.1\% and $12.6 \%$, respectively); as were alcohol abuse $(12.3 \%$ and $9.3 \%)$; illicit drug use $(8.5 \%$ and $4.5 \%)$; and bilateral disease (77.3\% and $62.8 \%$ ).

The proportion of unsuccessful outcome was 30.3\% in the primary resistance group and $46.7 \%$ in the acquired resistance group. LTFU was the most frequent unfavorable outcome in both groups at $12.3 \%$ among patients with primary resistance and $20.3 \%$ among those with acquired resistance; followed by death, with $10.8 \%$ in the primary resistance group and $16.1 \%$ in the acquired resistance group.

\section{Factors associated with treatment outcomes in bivariate models}

Table 2 shows the bivariate analyses of the factors associated with unsuccessful, LTFU, and death as outcomes for the group with primary resistance. In this group, the unsuccessful outcome was more likely in patients characterized by XDR-TB, illicit drug use, bilateral disease, less than eight years of schooling, unemployment, and comorbidities. LTFU was associated with being an inmate, illicit drug use, less than eight years of schooling, smoking, and cavitary disease.

The factors associated with the acquired resistance group are displayed in Table 3. Unsuccessful outcome was more likely in patients who were under 40 years old, with less than eight years of schooling, Afro-Brazilian, HIV-positive, illicit drug users, unemployed, with XDR-TB, and bilateral and cavitary disease. LTFU was associated with the following attributes: male, under 40 years old, less than eight years of schooling, Afro-Brazilian, HIV-positive, illicit drug use, alcohol abuse, smoking, unemployment, cavitary disease, and being an inmate. Having comorbidities and diabetes were protective factors for unsuccessful outcome and LTFU. Death was associated with the factors less than eight years of schooling, HIV-positive, comorbidities, bilateral disease, and XDR-TB. Culture conversion by the sixth month of treatment was a protective factor for all outcomes in both groups.

\section{Factors associated with treatment outcomes in the multivariate models}

Regarding unsuccessful outcome in the primary resistance group (Table 4), XDR-TB (ORa 12.2; 95\% CI 2.24-66.41) had a 12.2-fold higher odds than MDR-TB, while bilateral disease (ORa 1.92; 95\% CI 1.06-3.47) and the presence of comorbidities (ORa 2.06; 95\% CI 0.94-4.49) had nearly 2-fold higher odds. Having less than eight years of schooling was found to be associated with unsuccessful outcome (ORa 2.05; 95\% CI 1.13-3.72) and LTFU (ORa 2.30; 95\% CI 0.99-5.34).

As for the outcome LTFU in the primary resistance group, illicit drug users (ORa 3.38; 95\% CI 0.94-12.11) and smokers (ORa 2.91; 95\% CI 0.99-8.52) had approximately 3-fold higher odds. Being an inmate (ORa 8.00; 95\% CI 1.33-47.79) was found to have 8 -fold higher odds. Regarding death, unemployment (ORa 2.87; 95\% CI 1.17-7.04) was associated with 2.8-fold higher odds.

Regarding unsuccessful outcome in the acquired resistance group (Table 5), XDR-TB (ORa 5.43; 95\% CI 3.11-9.47) had 5.4fold higher odds than MDR-TB. Being under 40 years old, being Afro-Brazilian, and illicit drug use were found to be associated with unsuccessful and LTFU outcomes. HIV-positive and bilateral disease were found to be associated with unsuccessful and death outcomes. Having less than eight years of schooling was associated with all outcomes.

Unemployment was found to be associated only with the unsuccessful outcome (ORa 1.34; 95\% CI 0.98-1.81); and smoking (ORa 1.58; 95\% CI 1.02-2.45) only with LTFU. Comorbidity was associated with death (ORa 1.88; 95\% CI 1.22-2.88) and was a protective factor for LTFU (ORa 0.36; 95\% CI 0.21-0.62). In both groups, culture conversion by the sixth month was a protective factor for all outcomes, especially for unsuccessful and death outcomes. 
TABLE 1. Demographic and clinical characteristics of 1935 cases with acquired and 334 cases with primary MDR/XDR-TB notified in Rio de Janeiro State, 2000-2016

\begin{tabular}{|c|c|c|c|}
\hline Characteristics & $\begin{array}{l}\text { Primary resistance } \\
\qquad \%) N=334\end{array}$ & $\begin{array}{l}\text { Acquired resistance } \\
\text { (\%) } N=1935\end{array}$ & $p$-value * \\
\hline \multicolumn{4}{|c|}{ 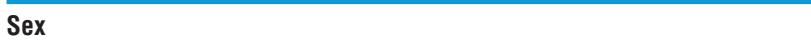 } \\
\hline Female & $155(46.4)$ & $648(33.5)$ & \multirow[t]{2}{*}{$<0.001$} \\
\hline Male & $179(53.6)$ & $1287(66.5)$ & \\
\hline \multicolumn{4}{|l|}{ Age range } \\
\hline $0-11$ & $2(0.6)$ & $2(0.1)$ & \multirow[t]{6}{*}{$<0.001$} \\
\hline $12-17$ & $13(3.9)$ & $30(1.5)$ & \\
\hline $18-24$ & $59(17.7)$ & $239(12.4)$ & \\
\hline $25-44$ & $134(40.1)$ & $992(51.3)$ & \\
\hline $45-64$ & $111(33.2)$ & $604(31.2)$ & \\
\hline$\geq 65$ & $15(4.5)$ & $68(3.5)$ & \\
\hline \multicolumn{4}{|l|}{ Ethnic group } \\
\hline Caucasian & $158(47.3)$ & $658(34.0)$ & \multirow[t]{3}{*}{$<0.001$} \\
\hline Afro-Brazilian & $170(50.9)$ & $1202(62.1)$ & \\
\hline Unknown & $6(1.8)$ & $74(3.8)$ & \\
\hline \multicolumn{4}{|l|}{$\begin{array}{l}\text { Years of } \\
\text { schooling }\end{array}$} \\
\hline None & $13(3.9)$ & $100(5.2)$ & \multirow[t]{6}{*}{$<0.001$} \\
\hline $1-3$ & $60(17.9)$ & $370(19.1)$ & \\
\hline $4-7$ & $109(32.7)$ & $770(39.8)$ & \\
\hline $8-11$ & $84(25.1)$ & $407(21.0)$ & \\
\hline$\geq 12$ & $42(12.6)$ & $124(6.4)$ & \\
\hline Unknown & $26(7.8)$ & $164(8.5)$ & \\
\hline \multicolumn{4}{|l|}{$\begin{array}{l}\text { Residence } \\
(n=2211)\end{array}$} \\
\hline Capital & 207 (65.5) & $1287(66.5)$ & \multirow[t]{2}{*}{0.003} \\
\hline $\begin{array}{l}\text { Other } \\
\text { municipalities }\end{array}$ & $109(34.5)$ & $648(33.5)$ & \\
\hline \multicolumn{4}{|l|}{ Site of disease } \\
\hline Extrapulmonary & $2(0.6)$ & $18(0.9)$ & \multirow[t]{3}{*}{0.010} \\
\hline Pulmonary & $321(96.1)$ & $1897(98.0)$ & \\
\hline Both & $11(3.3)$ & $20(1.1)$ & \\
\hline \multicolumn{4}{|l|}{$\begin{array}{l}\text { HIV status } \\
(n=2103)\end{array}$} \\
\hline Negative & $288(92.0)$ & $1648(92.1)$ & \multirow[t]{2}{*}{0.974} \\
\hline Positive & $25(8.0)$ & $142(7.9)$ & \\
\hline \multicolumn{4}{|l|}{ Other factors } \\
\hline Diabetes & 44 (13.2) & $175(9.0)$ & 0.018 \\
\hline Alcohol abuse & $31(9.3)$ & $238(12.3)$ & 0.937 \\
\hline Illicit drug use & $15(4.5)$ & $164(8.5)$ & 0.013 \\
\hline Smoking & $30(9.0)$ & $158(8.2)$ & 0.617 \\
\hline Inmate & $6(1.8)$ & $30(1.6)$ & 0.740 \\
\hline Unemployed & $42(12.6)$ & $331(17.1)$ & 0.039 \\
\hline Comorbidities $^{* *}$ & 48 (14.3) & $224(11.6)$ & 0.146 \\
\hline \multicolumn{4}{|l|}{$\begin{array}{l}\text { Chest radiography } \\
(n=2247)\end{array}$} \\
\hline Cavitation & $259(78.3)$ & $1560(81.4)$ & 0.175 \\
\hline Bilateral & $208(62.8)$ & $1485(77.3)$ & $<0.001$ \\
\hline \multicolumn{4}{|l|}{$\begin{array}{l}\text { Categories of drug } \\
\text { resistance }\end{array}$} \\
\hline MDR-TB & $324(97.0)$ & $1805(93.3)$ & 0.008 \\
\hline XDR-TB & $10(3.0)$ & $130(6.7)$ & \\
\hline \multicolumn{4}{|l|}{ Outcomes } \\
\hline Cured & $129(38.6)$ & $493(25.5)$ & $<0.001$ \\
\hline
\end{tabular}

TABLE 1. Demographic and clinical characteristics of 1935 cases with acquired and 334 cases with primary MDR/XDR-TB notified in Rio de Janeiro State, 2000-2016 (continued)

\begin{tabular}{lccc} 
Characteristics & $\begin{array}{c}\text { Primary resistance } \\
(\%) N=334\end{array}$ & $\begin{array}{c}\text { Acquired resistance } \\
(\%) N=1935\end{array}$ & $p$-value * \\
$\begin{array}{l}\text { Treatment } \\
\text { completed }\end{array}$ & $104(31.1)$ & $538(27.8)$ \\
Died & $36(10.8)$ & $311(16.1)$ \\
Loss to follow-up & $41(12.3)$ & $392(20.3)$ \\
Failed & $24(7.2)$ & $201(10.4)$ \\
\hline
\end{tabular}

OR, odds ratio; 95\% CI, 95\% confidence interval; TB, tuberculosis; MDR-TB, multidrug-resistant TB; XDR-TB, extensively drug-resistant TB; HIV, human immunodeficiency virus.

Comparison between primary and acquired MDR/XDR-TB using Pearson's chi-squared test

Except diabetes and HIV

Source: Prepared by the authors from the study results.

TABLE 2. Bivariate analysis: predictors of unsuccessful, lost to follow-up, and death outcomes among 334 primary MDR/ XDR-TB notified cases in Rio de Janeiro State, 2000-2016

\begin{tabular}{|c|c|c|c|}
\hline \multirow[t]{2}{*}{ Predictors } & Unsuccessful & Lost to follow-up & Death \\
\hline & $\begin{array}{c}\text { OR }(95 \% \mathrm{Cl}) \\
p \text {-value }\end{array}$ & $\begin{array}{c}\text { OR }(95 \% \mathrm{Cl}) \\
p \text {-value }\end{array}$ & $\begin{array}{c}\text { OR (95\% Cl) } \\
p \text {-value }\end{array}$ \\
\hline \multicolumn{4}{|l|}{ Sex } \\
\hline Female & 1.0 & 1.0 & 1.0 \\
\hline Male & $\begin{array}{c}0.88(0.55-1.41) \\
0.611\end{array}$ & $\begin{array}{c}1.12(0.58-2.16) \\
0.731\end{array}$ & $\begin{array}{c}0.66(0.33-1.32) \\
0.246\end{array}$ \\
\hline \multicolumn{4}{|l|}{$\geq 40$ years } \\
\hline Yes & 1.0 & 1.0 & 1.0 \\
\hline No & $\begin{array}{c}1.18(0.72-1.92) \\
0.508\end{array}$ & $\begin{array}{c}1.39(0.68-2.83) \\
0.360\end{array}$ & $\begin{array}{c}1.14(0.56-2.29) \\
0.707\end{array}$ \\
\hline \multicolumn{4}{|c|}{ Years of schooling } \\
\hline$\geq 8$ years & 1.0 & 1.0 & 1.0 \\
\hline$<8$ years & $\begin{array}{c}1.60(0.95-2.69) \\
0.074\end{array}$ & $\begin{array}{c}2.26(1.02-4.99) \\
0.043\end{array}$ & $\begin{array}{c}1.35(0.60-3.01) \\
0.461\end{array}$ \\
\hline \multicolumn{4}{|l|}{ Afro-Brazilian } \\
\hline No & 1.0 & 1.0 & 1.0 \\
\hline Yes & $\begin{array}{c}1.20(0.75-1.93) \\
0.439\end{array}$ & $\begin{array}{c}1.29(0.66-2.53) \\
0.445\end{array}$ & $\begin{array}{c}0.92(0.45-1.87) \\
0.822\end{array}$ \\
\hline \multicolumn{4}{|c|}{ HIV status $(n=313)$} \\
\hline Negative & 1.0 & 1.0 & 1.0 \\
\hline Positive & $\begin{array}{c}1.56(0.67-3.62) \\
0.295\end{array}$ & $\begin{array}{c}1.37(0.44-4.24) \\
0.578\end{array}$ & $\begin{array}{c}1.70(0.54-5.29) \\
0.359\end{array}$ \\
\hline \multicolumn{4}{|l|}{ Diabetes } \\
\hline No & 1.0 & 1.0 & 1.0 \\
\hline Yes & $\begin{array}{c}0.84(0.41-1.72) \\
0.646\end{array}$ & $\begin{array}{c}0.48(0.14-1.64) \\
0.246\end{array}$ & $\begin{array}{c}0.56(0.16-1.94) \\
0.369\end{array}$ \\
\hline \multicolumn{4}{|l|}{ Comorbidities* } \\
\hline No & 1.0 & 1.0 & 1.0 \\
\hline Yes & $\begin{array}{c}1.52(0.80-2.89) \\
0.197\end{array}$ & $\begin{array}{c}1.05(0.41-2.66) \\
0.912\end{array}$ & $\begin{array}{c}1.55(0.63-3.79) \\
0.330\end{array}$ \\
\hline \multicolumn{4}{|l|}{ Illicit drug use } \\
\hline No & 1.0 & 1.0 & 1.0 \\
\hline Yes & $\begin{array}{c}2.09(0.73-5.94) \\
0.165\end{array}$ & $\begin{array}{c}5.40(1.81-16.10) \\
0.002\end{array}$ & $(-)$ \\
\hline \multicolumn{4}{|l|}{ Alcohol abuse } \\
\hline No & 1.0 & 1.0 & 1.0 \\
\hline Yes & $\begin{array}{c}0.78(0.33-1.82) \\
0.573\end{array}$ & $\begin{array}{c}1.42(0.51-3.94) \\
0.494\end{array}$ & $\begin{array}{c}0.54(0.12-2.38) \\
0.421\end{array}$ \\
\hline
\end{tabular}

(continued) 
TABLE 2. Bivariate analysis: predictors of unsuccessful, lost to follow-up, and death outcomes among 334 primary MDR/ XDR-TB notified cases in Rio de Janeiro State, 2000-2016 (continued)

\begin{tabular}{|c|c|c|c|}
\hline \multirow[t]{2}{*}{ Predictors } & Unsuccessful & Lost to follow-up & Death \\
\hline & $\begin{array}{c}\text { OR (95\% Cl) } \\
p \text {-value }\end{array}$ & $\begin{array}{c}\text { OR }(95 \% \mathrm{Cl}) \\
p \text {-value }\end{array}$ & $\begin{array}{c}\text { OR (95\% CI) } \\
p \text {-value }\end{array}$ \\
\hline \multicolumn{4}{|l|}{ Smoking } \\
\hline No & 1.0 & 1.0 & 1.0 \\
\hline Yes & $\begin{array}{c}1.37(0.62-3.01) \\
0.423\end{array}$ & $\begin{array}{c}2.41(0.96-6.05) \\
0.060\end{array}$ & $\begin{array}{c}0.56(0.12-2.48) \\
0.452\end{array}$ \\
\hline \multicolumn{4}{|l|}{ Unemployed } \\
\hline No & 1.0 & 1.0 & 1.0 \\
\hline Yes & $\begin{array}{c}1.68(0.86-3.27) \\
0.125\end{array}$ & $\begin{array}{c}1.51(0.62-3.68) \\
0.357\end{array}$ & $\begin{array}{c}2.67(1.15-6.17) \\
0.021\end{array}$ \\
\hline \multicolumn{4}{|c|}{$\begin{array}{l}\text { Categories of drug } \\
\text { resistance }\end{array}$} \\
\hline MDR-TB & 1.0 & 1.0 & 1.0 \\
\hline XDR-TB & $\begin{array}{c}9.93(2.07-47.66) \\
0.004\end{array}$ & $(-)$ & $\begin{array}{c}2.13(0.43-10.45) \\
0.351\end{array}$ \\
\hline \multicolumn{4}{|c|}{$\begin{array}{l}\text { Six-month culture } \\
\text { conversion }\end{array}$} \\
\hline No & 1.0 & 1.0 & 1.0 \\
\hline Yes & $\begin{array}{c}0.19(0.11-0.34) \\
<0.001\end{array}$ & $\begin{array}{c}0.35(0.16-0.74) \\
0.006\end{array}$ & $\begin{array}{c}0.09(0.02-0.31) \\
<0.001\end{array}$ \\
\hline \multicolumn{4}{|c|}{$\begin{array}{l}\text { Chest radiography } \\
(n=324)\end{array}$} \\
\hline No cavitation & 1.0 & 1.0 & 1.0 \\
\hline Cavitation & $\begin{array}{c}1.40(0.77-2.54) \\
0.260\end{array}$ & $\begin{array}{c}2.82(0.97-8.19) \\
0.057\end{array}$ & $\begin{array}{c}0.68(0.31-1.50) \\
0.350\end{array}$ \\
\hline Unilateral & 1.0 & 1.0 & 1.0 \\
\hline Bilateral & $\begin{array}{c}1.82(1.09-3.02) \\
0.021\end{array}$ & $\begin{array}{c}1.30(0.65-2.63) \\
0.450\end{array}$ & $\begin{array}{c}1.60(0.74-3.45) \\
0.226\end{array}$ \\
\hline \multicolumn{4}{|l|}{ Inmate } \\
\hline No & 1.0 & 1.0 & 1.0 \\
\hline Yes & $\begin{array}{c}2.34(0.46-11.83) \\
0.301\end{array}$ & $\begin{array}{c}7.63(1.48-39.17) \\
0.015\end{array}$ & $(-)$ \\
\hline \multicolumn{4}{|c|}{ Treatment regimen } \\
\hline Standardized & 1.0 & 1.0 & 1.0 \\
\hline Individualized & $\begin{array}{c}1.30(0.78-2.14) \\
0.301\end{array}$ & $\begin{array}{c}0.94(0.46-1.94) \\
0.884\end{array}$ & $\begin{array}{c}0.71(0.32-1.58) \\
0.411\end{array}$ \\
\hline
\end{tabular}

\section{DISCUSSION}

The study showed that of the 2269 MDR-TB cases in the sample, $14.7 \%$ were primary resistance MDR-TB. Some studies conducted in different regions of Brazil show a variation in primary resistance MDR-TB of between $0.3 \%$ and $8.3 \%$ (11-14). However, these studies considered only patients who had no previous treatment record. In this study an expanded definition was adopted. Therefore, it includes cases of patients who start treatment with a basic regimen, progressing to failure and subsequent diagnosis of drug-resistant TB, or who undergo culture and sensitivity testing at the beginning of treatment and following tests for resistant TB. With this same definition, a study conducted in São Paulo with 156 MDR-TB patients found
TABLE 3. Bivariate analysis: predictors of unsuccessful, lost to follow-up, and death outcomes among 1935 acquired MDR/ XDR-TB notified cases in Rio de Janeiro State, 2000-2016

\begin{tabular}{|c|c|c|c|}
\hline \multirow[t]{2}{*}{ Predictors } & Unsuccessful & Lost to follow-up & Death \\
\hline & $\begin{array}{c}\text { OR (95\% Cl) } \\
p \text {-value }\end{array}$ & $\begin{array}{c}\text { OR (95\% Cl) } \\
p \text {-value }\end{array}$ & $\begin{array}{c}\text { OR (95\% Cl) } \\
p \text {-value }\end{array}$ \\
\hline \multicolumn{4}{|l|}{ Sex } \\
\hline Female & 1.0 & 1.0 & 1.0 \\
\hline Male & $\begin{array}{c}1.09(0.90-1.32) \\
0.347\end{array}$ & $\begin{array}{c}1.27(0.99-1.61) \\
0.051\end{array}$ & $\begin{array}{c}0.96(0.74-1.25) \\
0.808\end{array}$ \\
\hline \multicolumn{4}{|l|}{$\geq 40$ years } \\
\hline Yes & 1.0 & 1.0 & 1.0 \\
\hline No & $\begin{array}{c}1.30(1.08-1.56) \\
0.005\end{array}$ & $\begin{array}{c}1.58(1.26-2.00) \\
<0.001\end{array}$ & $\begin{array}{c}0.92(0.72-1.18) \\
0.558\end{array}$ \\
\hline \multicolumn{4}{|c|}{ Years of schooling } \\
\hline$\geq 8$ years & 1.0 & 1.0 & 1.0 \\
\hline$<8$ years & $\begin{array}{c}1.72(1.40-2.12) \\
<0.001\end{array}$ & $\begin{array}{c}1.73(1.31-2.29) \\
<0.001\end{array}$ & $\begin{array}{c}1.68(1.23-2.29) \\
0.001\end{array}$ \\
\hline \multicolumn{4}{|l|}{ Afro-Brazilian } \\
\hline No & 1.0 & 1.0 & 1.0 \\
\hline Yes & $\begin{array}{c}1.36(1.12-1.65) \\
0.001\end{array}$ & $\begin{array}{c}1.64(1.28-2.11) \\
<0.001\end{array}$ & $\begin{array}{c}0.99(0.76-1.28) \\
0.968\end{array}$ \\
\hline \multicolumn{4}{|c|}{ HIV status $(n=1790)$} \\
\hline Negative & 1.0 & 1.0 & 1.0 \\
\hline Positive & $\begin{array}{c}1.42(1.01-2.01) \\
0.042\end{array}$ & $\begin{array}{c}1.47(0.99-2.19) \\
0.051\end{array}$ & $\begin{array}{c}1.45(0.95-2.21) \\
0.078\end{array}$ \\
\hline \multicolumn{4}{|l|}{ Diabetes } \\
\hline No & 1.0 & 1.0 & 1.0 \\
\hline Yes & $\begin{array}{c}0.72(0.52-0.99) \\
0.043\end{array}$ & $\begin{array}{c}0.39(0.23-0.66) \\
<0.001\end{array}$ & $\begin{array}{c}0.77(0.49-1.22) \\
0.270\end{array}$ \\
\hline \multicolumn{4}{|l|}{ Comorbidities* } \\
\hline No & 1.0 & 1.0 & 1.0 \\
\hline Yes & $\begin{array}{c}0.82(0.61-1.08) \\
0.170\end{array}$ & $\begin{array}{c}0.41(0.26-0.65) \\
<0.001\end{array}$ & $\begin{array}{c}1.54(1.09-2.18) \\
0.012\end{array}$ \\
\hline \multicolumn{4}{|l|}{ Illicit drug use } \\
\hline No & 1.0 & 1.0 & 1.0 \\
\hline Yes & $\begin{array}{c}1.87(1.35-2.60) \\
<0.001\end{array}$ & $\begin{array}{c}2.89(2.07-4.05) \\
<0.001\end{array}$ & $\begin{array}{c}0.79(0.49-1.26) \\
0.334\end{array}$ \\
\hline \multicolumn{4}{|l|}{ Alcohol abuse } \\
\hline No & 1.0 & 1.0 & 1.0 \\
\hline Yes & $\begin{array}{c}1.20(0.92-1.58) \\
0.174\end{array}$ & $\begin{array}{c}1.53(1.12-2.09) \\
0.007\end{array}$ & $\begin{array}{c}1.06(0.73-1.52) \\
0.742\end{array}$ \\
\hline \multicolumn{4}{|l|}{ Smoking } \\
\hline No & 1.0 & 1.0 & 1.0 \\
\hline Yes & $\begin{array}{c}1.12(0.81-1.55) \\
0.486\end{array}$ & $\begin{array}{c}1.69(1.18-2.44) \\
0.004\end{array}$ & $\begin{array}{c}0.48(0.27-0.85) \\
0.012\end{array}$ \\
\hline \multicolumn{4}{|l|}{ Unemployed } \\
\hline No & 1.0 & 1.0 & 1.0 \\
\hline Yes & $\begin{array}{c}1.55(1.22-1.97) \\
<0.001\end{array}$ & $\begin{array}{c}1.51(1.15-1.99) \\
0.003\end{array}$ & $\begin{array}{c}0.96(0.69-1.33) \\
0.844\end{array}$ \\
\hline \multicolumn{4}{|c|}{$\begin{array}{l}\text { Categories of drug } \\
\text { resistance }\end{array}$} \\
\hline MDR-TB & 1.0 & 1.0 & 1.0 \\
\hline XDR-TB & $\begin{aligned} 5.57 & (3.54-8.76) \\
& <0.001\end{aligned}$ & $\begin{array}{c}0.65(0.39-1.08) \\
0.100\end{array}$ & $\begin{array}{c}2.51(1.69-3.73) \\
<0.001\end{array}$ \\
\hline \multicolumn{4}{|c|}{$\begin{array}{l}\text { Six-month culture } \\
\text { conversion }\end{array}$} \\
\hline No & 1.0 & 1.0 & 1.0 \\
\hline Yes & $\begin{array}{c}0.18(0.15-0.23) \\
<0.001\end{array}$ & $\begin{array}{c}0.44(0.34-0.58) \\
<0.001\end{array}$ & $\begin{array}{c}0.11(0.06-0.17) \\
<0.001\end{array}$ \\
\hline
\end{tabular}


TABLE 3. Bivariate analysis: predictors of unsuccessful, lost to follow-up, and death outcomes among 1935 acquired MDR/ XDR-TB notified cases in Rio de Janeiro State, 2000-2016 (continued)

\begin{tabular}{|c|c|c|c|}
\hline \multirow[t]{2}{*}{ Predictors } & Unsuccessful & Lost to follow-up & Death \\
\hline & $\begin{array}{c}\text { OR (95\% Cl) } \\
p \text {-value }\end{array}$ & $\begin{array}{c}\text { OR }(95 \% \mathrm{Cl}) \\
p \text {-value }\end{array}$ & $\begin{array}{c}\text { OR (95\% Cl) } \\
p \text {-value }\end{array}$ \\
\hline \multicolumn{4}{|c|}{$\begin{array}{l}\text { Chest radiography } \\
(n=1923)\end{array}$} \\
\hline No cavitation & 1.0 & 1.0 & 1.0 \\
\hline Cavitation & $\begin{array}{c}1.63(1.28-2.07) \\
<0.001\end{array}$ & $\begin{array}{c}1.36(1.00-1.86) \\
0.047\end{array}$ & $\begin{array}{c}1.03(0.75-1.41 \\
0.842\end{array}$ \\
\hline Unilateral & 1.0 & 1.0 & 1.0 \\
\hline Bilateral & $\begin{array}{c}1.93(1.54-2.42) \\
<0.001\end{array}$ & $\begin{array}{c}1.01(0.77-1.32) \\
0.926\end{array}$ & $\begin{array}{c}2.66(1.83-3.87 \\
<0.001\end{array}$ \\
\hline \multicolumn{4}{|l|}{ Inmate } \\
\hline No & 1.0 & 1.0 & 1.0 \\
\hline Yes & $\begin{array}{c}0.99(0.48-2.05) \\
0.995\end{array}$ & $\begin{array}{c}2.31(1.09-4.90) \\
0.028\end{array}$ & $\begin{array}{c}0.36(0.08-1.55 \\
0.175\end{array}$ \\
\hline \multicolumn{4}{|c|}{ Treatment regimen } \\
\hline Standardized & 1.0 & 1.0 & 1.0 \\
\hline Individualized & $\begin{array}{c}1.55(0.73-0.89) \\
<0.001\end{array}$ & $\begin{array}{c}1.02(0.81-1.29) \\
0.834\end{array}$ & $\begin{array}{c}0.97(0.75-1.25 \\
0.817\end{array}$ \\
\hline
\end{tabular}

OR, odds ratio; $95 \% \mathrm{Cl}, 95 \%$ confidence interval; TB, tuberculosis; MDR-TB, multidrug-resistant TB XDR-TB, extensively drug-resistant TB; HIV, human immunodeficiency virus.

"Except diabetes and HIV

Source: Prepared by the authors from the study results.

$36 \%$ of primary resistance cases. Note that only $5 \%$ of patients reported not having undergone previous treatment for TB (15). Another study conducted in RJ showed that approximately $30 \%$ of XDR-TB cases had no previous treatment for multidrug resistance. This suggests that a high proportion of cases of XDR-TB is due to primary infection in RJ (16). It may also mean that patients with primary resistance can begin basic TB treatment, with the diagnosis delayed by the absence of initial drug resistance detection.

Proportionally, the primary resistance group had more females, Caucasians, younger patients, schooling, diabetes, and other comorbidities than the acquired group. A study conducted in China found that women had a 1.64-fold increased risk of primary MDR-TB (17). In Peru, a study conducted in the urban area of Lima showed that patients with primary resistance were younger and had better schooling. These results highlight that primary MDR-TB cases constitute a different reservoir of transmission, less related to lower socioeconomic groups and more related to other chronic diseases associated with higher TB acquisition (18).

Furthermore, studies in Brazil, India, and South Africa also showed a high incidence rate of MDR-TB among contacts, mainly among children and young people (19-21). The characteristics of cases of the primary resistance group differ from those of the acquired resistance group. Probably, the former is likely to be composed of people who spend more time in contact with infected people, like in the household (15). More recently, epidemiology and genetics studies with MDR-TB patients in China identified 93 clusters, in which $69 \%$ of the cases had epidemiological links with people they knew. The most common relationships between cases were social, such as living in the
TABLE 4. Multivariate analysis: predictors of unsuccessful, lost to follow-up, and death outcomes among 334 primary MDR/XDR-TB notified cases in Rio de Janeiro State, 2000-2016

\begin{tabular}{|c|c|c|c|}
\hline \multirow[t]{2}{*}{ Predictors } & Unsuccessful & Lost to follow-up & Death \\
\hline & $\begin{array}{c}\text { ORa }(95 \% \mathrm{Cl}) \\
p \text {-value }\end{array}$ & $\begin{array}{c}\text { ORa }(95 \% \mathrm{Cl}) \\
p \text {-value }\end{array}$ & $\begin{array}{c}\text { ORa }(95 \% \mathrm{Cl}) \\
p \text {-value }\end{array}$ \\
\hline \multicolumn{4}{|c|}{ Years of schooling } \\
\hline$\geq 8$ years & 1.0 & 1.0 & \\
\hline$<8$ years & $\begin{array}{c}2.05(1.13-3.72) \\
0.018\end{array}$ & $\begin{array}{c}2.30(0.99-5.34) \\
0.051\end{array}$ & \\
\hline \multicolumn{4}{|l|}{ Unemployment } \\
\hline No & & & 1.0 \\
\hline Yes & & & $\begin{array}{c}2.87(1.17-7.04) \\
0.021\end{array}$ \\
\hline \multicolumn{4}{|l|}{ Drug use } \\
\hline No & & 1.0 & \\
\hline Yes & & $\begin{array}{c}3.38(0.94-12.11) \\
0.061\end{array}$ & \\
\hline \multicolumn{4}{|l|}{ Smoking } \\
\hline No & & 1.0 & \\
\hline Yes & & $\begin{array}{c}2.91(0.99-8.52) \\
0.051\end{array}$ & \\
\hline \multicolumn{4}{|c|}{$\begin{array}{l}\text { Categories of drug } \\
\text { resistance }\end{array}$} \\
\hline MDR-TB & 1.0 & & \\
\hline XDR-TB & $\begin{array}{c}12.2(2.24-66.41) \\
0.004\end{array}$ & & \\
\hline \multicolumn{4}{|c|}{ Chest radiography } \\
\hline Unilateral & 1.0 & & \\
\hline Bilateral & $\begin{array}{c}1.92(1.06-3.47) \\
0.030\end{array}$ & & \\
\hline \multicolumn{4}{|l|}{ Comorbidities ${ }^{\star}$} \\
\hline No & 1.0 & & \\
\hline Yes & $\begin{array}{c}2.06(0.94-4.49) \\
0.068\end{array}$ & & \\
\hline \multicolumn{4}{|c|}{$\begin{array}{l}\text { Six-month culture } \\
\text { conversion }\end{array}$} \\
\hline No & 1.0 & 1.0 & 1.0 \\
\hline Yes & $\begin{array}{c}0.19(0.10-0.35) \\
<0.001\end{array}$ & $\begin{array}{c}0.36(0.16-0.81) \\
0.015\end{array}$ & $\begin{array}{c}0.08(0.25-0.28) \\
<0.001\end{array}$ \\
\hline \multicolumn{4}{|l|}{ Inmate } \\
\hline No & & 1.0 & \\
\hline Yes & & $\begin{array}{c}8.00(1.33-47.79) \\
0.023\end{array}$ & \\
\hline
\end{tabular}

ORa, adjusted odds ratio; 95\% Cl, 95\% confidence interval; TB, tuberculosis; MDR-TB, multidrug-resistant TB XDR-TB, extensively drug-resistant TB. Except diabetes and HIV

Source: Prepared by the authors from the study results.

same residential complex, in the same street, or attending the same public places (22).

Socioeconomic factors are connected to the health-disease process and are significant predictors of the outcome of TB. Regarding treatment outcome, the cases with primary MDR-TB had a higher therapeutic success rate than the cases with acquired resistance $(69.7 \%$ and $53.3 \%$, respectively). The superior performance of the primary resistance group was also observed in another study that showed 6.3-fold higher odds of cure (15). Higher socioeconomic status is associated with a nearly 3-fold increased risk of primary MDR-TB as compared 
TABLE 5. Multivariate analysis: predictors of unsuccessful, lost to follow-up, and death outcomes among 1935 acquired MDR/XDR-TB notified cases in Rio de Janeiro State, 2000-2016

\begin{tabular}{|c|c|c|c|}
\hline \multirow[t]{2}{*}{ Predictors } & \multicolumn{3}{|c|}{ Lost to follow-up } \\
\hline & $\begin{array}{c}\text { ORa }(95 \% \mathrm{Cl}) \\
p \text {-value }\end{array}$ & $\begin{array}{c}\text { ORa }(95 \% \mathrm{Cl}) \\
p \text {-value }\end{array}$ & $\begin{array}{c}\text { ORa }(95 \% \mathrm{Cl}) \\
p \text {-value }\end{array}$ \\
\hline \multicolumn{4}{|l|}{$\geq 40$ years } \\
\hline Yes & 1.0 & 1.0 & \\
\hline No & $\begin{array}{c}1.24(0.98-1.57) \\
0.068\end{array}$ & $\begin{array}{c}1.55(1.19-2.01) \\
0.001\end{array}$ & \\
\hline \multicolumn{4}{|c|}{ Years of schooling } \\
\hline$\geq 8$ years & 1.0 & 1.0 & 1.0 \\
\hline$<8$ years & $\begin{array}{c}1.61(1.23-2.10) \\
<0.001\end{array}$ & $\begin{array}{c}1.52(1.12-2.05) \\
0.006\end{array}$ & $\begin{array}{c}1.50(1.05-2.15) \\
0.023\end{array}$ \\
\hline \multicolumn{4}{|l|}{ Afro-Brazilian } \\
\hline No & 1.0 & 1.0 & \\
\hline Yes & $\begin{array}{c}1.42(1.14-1.86) \\
0.002\end{array}$ & $\begin{array}{c}1.67(1.26-2.22) \\
<0.001\end{array}$ & \\
\hline \multicolumn{4}{|l|}{ Unemployment } \\
\hline No & 1.0 & & \\
\hline Yes & $\begin{array}{c}1.34(0.98-1.81) \\
0.058\end{array}$ & & \\
\hline \multicolumn{4}{|l|}{ Illicit drug use } \\
\hline No & 1.0 & 1.0 & \\
\hline Yes & $\begin{array}{c}1.74(1.11-2.74) \\
<0.001\end{array}$ & $\begin{array}{c}2.34(1.56-3.53) \\
<0.001\end{array}$ & \\
\hline \multicolumn{4}{|l|}{ Smoking } \\
\hline No & & 1.0 & \\
\hline Yes & & $\begin{array}{c}1.58(1.02-2.45) \\
<0.001\end{array}$ & \\
\hline \multicolumn{4}{|l|}{$\begin{array}{l}\text { HIV status } \\
(n=1790)\end{array}$} \\
\hline Negative & 1.0 & & 1.0 \\
\hline Positive & $\begin{array}{c}1.57(1.00-2.48) \\
0.048\end{array}$ & & $\begin{array}{c}1.61(0.98-2.65) \\
0.057\end{array}$ \\
\hline \multicolumn{4}{|c|}{$\begin{array}{l}\text { Categories of drug } \\
\text { resistance }\end{array}$} \\
\hline MDR-TB & 1.0 & & 1.0 \\
\hline XDR-TB & $\begin{array}{c}5.43(3.11-9.47) \\
<0.001\end{array}$ & & $\begin{array}{c}2.26(1.42-3.62) \\
0.001\end{array}$ \\
\hline \multicolumn{4}{|c|}{ Chest radiography } \\
\hline Unilateral & 1.0 & & 1.0 \\
\hline Bilateral & $\begin{array}{c}2.20(1.66-2.93) \\
<0.001\end{array}$ & & $\begin{array}{cl}2.90 & (1.86-4.51) \\
& <0.001\end{array}$ \\
\hline \multicolumn{4}{|l|}{ Comorbidities ${ }^{\star}$} \\
\hline No & & 1.0 & 1.0 \\
\hline Yes & & $\begin{array}{c}0.36(0.21-0.62) \\
<0.001\end{array}$ & $\begin{array}{c}1.88(1.22-2.88) \\
0.004\end{array}$ \\
\hline \multicolumn{4}{|c|}{$\begin{array}{l}\text { Six-month culture } \\
\text { conversion }\end{array}$} \\
\hline No & 1.0 & 1.0 & 1.0 \\
\hline Yes & $\begin{array}{c}0.17(0.13-0.23) \\
<0.001\end{array}$ & $\begin{array}{c}0.48(0.36-0.64) \\
<0.001\end{array}$ & $\begin{array}{c}0.08(0.04-0.14) \\
<0.001\end{array}$ \\
\hline
\end{tabular}

ORa, adjusted odds ratio; 95\% Cl, 95\% confidence interval; TB, tuberculosis; MDR-TB, multidrug-resistant TB; XDR-TB, extensively drug-resistant TB; HIV, human immunodeficiency virus.

Source: Prepared by the authors from the study results.

with acquired resistance and, conversely, lower socioeconomic status is associated with increased risk of acquired MDR-TB (18).

In this study, low schooling and unhealthy behavior factors (illicit drug use, smoking) were associated with the unsuccessful outcome or LTFU in both groups. Low schooling may restrict understanding of the disease, leading to errors in treatment mainly due to the inappropriate use of medicines. Low schooling also makes it difficult to comply with routines and performance tests, contributing to unfavorable results (23). Unemployment, being Afro-Brazilian, illicit drug use, smoking, and being under 40 years old were associated with LTFU among acquired resistance cases. Unemployment was the only factor associated with death among primary MDR-TB cases. Although many studies show that socioeconomic or behavioral factors are a risk for MDR-TB, these factors may also indicate poor access to health services (24).

Among the acquired MDR-TB group, death was associated with less than eight years of schooling, being HIV-positive, bilateral disease, comorbidity, and XDR-TB, as found by other authors (25-28). HIV-positive patients had a 1.6-fold higher rate of mortality than those who were HIV-negative. However, HIV-positive was only associated with the acquired resistance group. One hypothesis is that low adherence to TB treatment and the use of intermittent treatment regimens-factors associated with worse outcomes and which are frequent in this group (28) - also occur in antiretroviral treatment.

Being an inmate was found to be a variable strongly associated with LTFU in the primary resistance group. In 2012, a study conducted in a prison in RJ showed that $83 \%$ of MTB strains belonged to one of the 13 identified clusters, suggesting high intrainstitutional transmission (29). In the same year, RJ had around 20000 inmates distributed across 37 prison units (29). LTFU, in turn, may be related to scarce interaction between prison health care and health care services coordinated by municipal and state authorities. Such interaction decreases the probability that medication is taken. According to Sánchez and Larouzé, people in jail tend to reject impositions beyond those already suffered. Moreover, the coercive strategies often employed to ensure the intake of medication end up having limited effectiveness (30). These results confirm the recent statements issued by NTP-MoH, according to which prisons have favorable conditions for amplifying TB or primary MDR-TB in the general population and are reservoirs of the disease (4).

Moreover, having XDR-TB was more strongly associated with the unsuccessful outcome in the primary MDR-TB group than in the acquired MDR-TB group (ORa 12.2 and 5.43, respectively). This fact may reflect the delay in the diagnosis of drug resistance and an increase in the severity of the cases, as drug sensitivity tests for second-line drugs are only performed in patients known to have MDR-TB (31).

Having comorbidities was a protective factor for LTFU among the acquired resistance group. Perhaps this is because tertiary care provided a better resolution for these patients' comorbidities and, therefore, they adhered to treatment more frequently (16).

Finally, as other studies have shown, culture conversion by six months was a protective factor in both groups, being a good predictor for treatment success $(32,33)$.

For a long time, the WHO recommendation for the detection of drug resistance targeted patients with a history of TB treatment, not considering patients with primary drug resistance (34). Although the Xpert MTB/RIF molecular test has been available since 2014 , only $30 \%$ to $40 \%$ of TB patients under treatment have been analyzed using the molecular test (4). Although the NTP-MoH recommendation is that culture 
achievement and drug sensitivity testing have to be performed for all retreatment cases, in RJ, culture was performed in only $19.8 \%$ of patients in 2018 (6). Among the cases with a positive culture, drug sensitivity testing was performed in only $58.6 \%$. Moreover, only $34.2 \%$ of the contacts of new cases of pulmonary TB with laboratory confirmation were examined (4). This poor performance can contribute to increasing the transmission of primary MDR-TB, therefore driving the spread of drug resistance, as has already been observed in countries such as China, India, and South Africa (24).

One of the limitations of this study was the impossibility of using drug resistance as an independent variable, due to the low number of DSTs reported for first- and second-line drugs. Another limitation is that until 2015, in the SITETB database, variables related to diabetes, comorbidities, illicit drug use, alcohol abuse, and smoking when classified as "no" can also mean lack of information. There is also no standardization for the classification of alcohol abuse, smoking, and mental health disorders. At any rate, the statistical findings reported here are robust due to the large sample size on which they are based.

In conclusion, a primary resistance of $14.7 \%$ among MDR-TB cases was found in RJ in 2000-2016. The cases with primary MDR-TB had a higher therapeutic success rate than the cases with acquired resistance. Differences between the groups indicate that primary MDR-TB cases constitute a different reservoir of transmission, less related to lower socioeconomic groups and more related to other chronic diseases associated with higher TB acquisition. Moreover, the poor results observed in RJ can contribute to increasing the transmission of primary MDR-TB, therefore driving the spread of drug resistance. Recommendations include (1) improving the use of Xpert MTB/RIF as the first approach to a presumed TB patient, (2) increasing the early diagnosis of drug resistance, and (3) improving TB contact tracing.

Author contributions. MB and AK designed the study. MB conducted the analyses and drafted the manuscript. AK critically revised it. Both authors reviewed and approved the final version.

\section{Conflict of interest. None declared.}

Disclaimer. Authors hold sole responsibility for the views expressed in the manuscript, which may not necessarily reflect the opinion or policy of the RPSP/PAJPH or the Pan American Health Organization (PAHO).

\section{REFERENCES}

1. World Health Organization. Global tuberculosis report 2020. Geneva: WHO; 2020.

2. Li X, Lu W, Zu R, Zhu L, Yang H, Chen C, et al. Comparing risk factors for primary multidrug-resistant tuberculosis and primary drug-susceptible tuberculosis in Jiangsu Province, China : a matched-pairs case-control study. Am J Med Hyg. 2015;92(2):280-5.

3. Kritski AL. Emergência de tuberculose resistente: renovado desafio. J Bras Pneumol. 2010;36(2):157-8.

4. Ministério da Saúde Brasil. Brasil Livre da Tuberculose: evolução dos cenários epidemiológicos e operacionais da doença. Bol Epidemiológico [Internet]. 2019;50(9):1-18. Available from: http:// portalarquivos2.saude.gov.br/images/pdf/2019/marco/22/2019009.pdf

5. Instituto Brasileiro de Geografia e Estatística [accessed May 2020]. Available from: https://cidades.ibge.gov.br/brasil/rj/ rio-de-janeiro/panorama

6. Rocha MS, Bartholomay P, Cavalcante MV, Medeiros FC de, Codenotti SB, Pelissari DM, et al. Sistema de Informação de Agravos de Notificação (SINAN): principais características da notificação e da análise de dados relacionada à tuberculose. Epidemiol Serv Saude. 2020;29(1):e2019017.

7. Tourinho BD, Oliveira PB, Silva GDM da, Rocha MS, Penna EQA de A, Pércio J. Evaluation of the Drug-Resistant Tuberculosis Surveillance System, Brazil, 2013-2017. Epidemiol Serv Saude. 2020;29(1):e2019190.

8. Departamento de Vigilância Epidemiológica, Secretaria de Vigilância em Saúde, Ministério da Saúde. Sistema de Informação de Tratamentos Especiais da Tuberculose - Manual do usuário. Brasília: Ministério da Saúde; 2012. Available from: http:/ / sitetb.saude.gov. br/download/sitetb_notificar_caso.v03_11_2011.pdf

9. Wilhelm D, Rodrigues MV, Nakata PT, Godoy SDC, Blatt CR. Descentralização do acesso ao Sistema de Informações de Tratamentos Especiais em Tuberculose. Rev Baiana Enfermagem [Internet]. 2018;32:1-10.

10. World Health Organization. Companion handbook to the WHO guidelines for the programmatic management of drug-resistant tuberculosis. Geneva: WHO; 2014.

11. Souza MB, Antunes CM, Garcia GF. Multidrug-resistant Mycobacterium tuberculosis at a referral center for infectious diseases in the state of Minas Gerais, Brazil: sensitivity profile and related risk factors. J Bras Pneumol. 2006;32(5):430-7.

12. Garrido MS, Ramasawmy R, Perez-Porcuna TM, Zaranza E, Talhari AC, Martinez-Espinosa MF, et al. Primary drug resistance among pulmonary treatment-naïve tuberculosis patients in Amazonas State, Brazil. Int J Tuberc Lung Dis. 2014;18(5):559-63.

13. Marques M, Cunha EAT, Ruffino-Netto A, Andrade SMO. Perfil de resistência de Mycobacterium tuberculosis no Estado de Mato Grosso do Sul, 2000-2006. J Bras Pneumol. 2010;36(2):224-31.

14. Baliza M, Bach AH, Queiroz GL, Melo IC, Carneiro MM, Albuquerque MFPM de, et al. High frequency of resistance to the drugs isoniazid and rifampicin among tuberculosis cases in the city of Cabo de Santo Agostinho, an urban area in northeastern Brazil. Rev Soc Bras Med Trop. 2008;41:11-6.

15. Savioli1 MTG, Morrone N, Santoro I. Primary bacillary resistance in multidrug-resistant tuberculosis and predictive factors associated with cure at a referral center in São Paulo, Brazil. J Bras Pneumol. 2019;45(2):e20180075.

16. Bhering M, Duarte R, Kritski A. Predictive factors for unfavourable treatment in MDR-TB and XDR-TB patients in Rio de Janeiro State, Brazil, 2000-2016. PLOS One. 2019;14(11):e0218299.

17. Wang SF, Zhou Y, Pang Yu, Zheng HW, Zhao YL. Prevalence and risk factors of primary drug-resistant tuberculosis in China. Biomed Environ Sci. 2016;29(2):91-8.

18. Odone A, Calderon R, Becerra MC, Zhang Z, Contreras CC, Yataco $\mathrm{R}$, et al. Acquired and transmitted multidrug resistant tuberculosis: the role of social determinants. PLOS One. 2016;11(1):e0146642.

19. Teixeira L, Perkins MD, Johnson JL, Keller R, Palaci M, Dettoni VV, et al. Infection and disease among household contacts of patients with multidrug-resistant tuberculosis. Int J Tuberc Lung Dis. 2001;5(4):321-8.

20. Singla N, Singla R, Jain G, Habib L, Behera D. Tuberculosis among household contacts of multidrug-resistant tuberculosis patients in Delhi, India. Int J Tuberc Lung Dis. 2011;15(10):1326-30.

21. Vella V, Racalbuto V, Guerra R, Marra C, Moll A, Mhlanga Z, et al. Household contact investigation of multidrug-resistant and extensively drug-resistant tuberculosis in a high HIV prevalence setting. Int J Tuberc Lung Dis. 2011;15(9):1170-5. 
22. Yang C, Luo T, Shen X, Wu J, Gan M, Xu P, et al. Transmission of multidrug-resistant Mycobacterium tuberculosis in Shanghai, China : a retrospective observational study using whole-genome sequencing and epidemiological. Lancet Infect Dis. 2017;17(3):275-84.

23. Paula HC de, Aguiar AC de. $\mathrm{O}$ abandono do tratamento da tuberculose na estratégia saúde da família: estudo qualitativo em uma área programática do Rio de Janeiro. Rev Baiana Saude Publica. 2013;192-204.

24. Dheda K, Gumbo T, Maartens G, Dooley KE, McNerney R, Murray $\mathrm{M}$, et al. The epidemiology, pathogenesis, transmission, diagnosis, and management of multidrug-resistant, extensively drug-resistant, and incurable tuberculosis. Lancet Respir Med. 2017;5:291-360.

25. Gayoso R, Dalcolmo M, Braga JU, Barreira D. Predictors of mortality in multidrug-resistant tuberculosis patients from Brazilian reference centers, 2005 to 2012. Brazilian J Infect Dis. 2018;22(4):305-10.

26. Kurbatova EV, Taylor A, Gammino VM, Bayona J, Becerra M, Danilovitz M, et al. Predictors of poor outcomes among patients treated for multidrug-resistant tuberculosis at DOTS-plus projects. Tuberculosis (Edinb). 2012;92(5):397-403.

27. Kim DH, Kim HJ, Park SK, Kong SJ, Kim YS, Kim TH, et al. Treatment outcomes and survival based on drug resistance patterns in multidrug-resistant tuberculosis. Am J Respir Crit Care Med. 2010;182(1):113-9.

28. Wells CD, Cegielski JP, Nelson LJ, Laserson KF, Holtz TH, Finlay A, et al. HIV infection and multidrug-resistant tuberculosis: the perfect storm. J Infect Dis. 2007;196(s1):S86-107.

29. Sánchez A, Huber FD, Massari V, Barreto A, Camacho LAB, Cesconi V, et al. Extensive Mycobacterium tuberculosis circulation in a highly endemic prison and the need for urgent environmental interventions. Epidemiol Infect. 2012;140(10):1853-61.

30. Sánchez A, Larouzé B. Controle da tuberculose nas prisões, da pesquisa à ação: a experiência do Rio de Janeiro, Brasil. Cien Saude Colet. 2016;21(7):2071-80.

31. Departamento de Vigilância Epidemiológica, Secretaria de Vigilância em Saúde, Ministério da Saúde. Manual de recomendações para controle da tuberculose no Brasil. Brasília: Ministério da Saúde; 2011.

32. Qazi F, Khan U, Khowaja S, Javaid M, Ahmed A, Salahuddin N, et al. Predictors of delayed culture conversion in patients treated for multidrug-resistant tuberculosis in Pakistan. Int J Tuberc Lung Dis. 2011;15(11):1556-9.

33. Kurbatova EV, Cegielski JP, Lienhardt C, Akksilp R, Bayona J, Becerra MC, et al. Sputum culture conversion as a prognostic marker for end-of-treatment outcome in patients with multidrug-resistant tuberculosis: a secondary analysis of data from two observational cohort studies. Lancet Respir Med. 2015;3(3):201-9.

34. World Health Organization. Guidelines for the programmatic management of drug-resistant tuberculosis. Geneva: WHO; 2008.

Manuscript received on 10 January 2020. Revised version accepted for publication on 22 June 2020

\section{Tuberculosis multirresistente primaria o adquirida: factores predictivos de resultados desfavorables del tratamiento en Rio de Janeiro entre el 2000 y 2016}

\section{RESUMEN}

Palabras clave
Objetivo. Determinar cuáles son los factores clínicos o demográficos asociados con resultados desfavorables del tratamiento en pacientes con tuberculosis multirresistente (TB-MDR) primaria o adquirida en el estado de Rio de Janeiro.

Métodos. Estudio retrospectivo de cohorte con datos de 2269 casos de TB-MDR entre el 2000 y el 2016 . Se analizaron los factores asociados con resultados desfavorables, pacientes perdidos en el seguimiento o muerte en pacientes con resistencia primaria o adquirida mediante modelos de regresión bifactorial y multifactorial.

Resultados. En los casos de TB-MDR, la resistencia primaria fue de 14,7\%. La proporción de resultados desfavorables fue de $30,3 \%$ en el grupo con resistencia primaria y de $46,7 \%$ en el grupo con resistencia adquirida. Las diferencias en las características demográficas y clínicas de los dos grupos fueron significativas. Proporcionalmente, el grupo con resistencia primaria tuvo más casos en las mujeres $(46,4 \%$ frente a $33,5 \%$ en el grupo con resistencia adquirida), las personas caucásicas (47,3\% frente a $34 \%$ ) y en aquellas personas con 8 o más años de escolarización (37,7\% frente a 27,4\%). Los pacientes con tuberculosis extensamente resistente tenían 12,2 veces más probabilidades de tener un resultado desfavorable que los pacientes con TB-MDR. La comorbilidad fue 2 veces mayor en el grupo con resistencia primaria. El grupo con TB-MDR adquirida tenía 5,43 veces más probabilidades de tener tuberculosis extremadamente resistente. En ambos grupos, se asoció la enfermedad bilateral y menos de 8 años de escolarización con resultados desfavorables. En el grupo con resistencia primaria, las probabilidades de perder al paciente en el seguimiento fueron 8 veces mayores en los presidiarios. La conversión del cultivo a los seis meses fue un factor protector en todos los resultados.

Conclusiones. Los casos de resistencia primaria de TB-MDR constituyen un reservorio de transmisión diferente, que está relacionado con otras enfermedades crónicas asociadas con una mayor adquisición de tuberculosis. Los resultados insatisfactorios observados en el estado de Rio de Janeiro pueden contribuir a aumentar la transmisión de la TB-MDR primaria, y por lo tanto favorecer la farmacorresistencia. 


\section{Tuberculose multirresistente primária e adquirida: preditores de desfechos desfavoráveis do tratamento no Rio de Janeiro, 2000-2016}

RESUMO

Objetivo. Identificar fatores demográficos e clínicos associados a desfechos desfavoráveis do tratamento em pacientes com tuberculose multirresistente primária e adquirida no Estado do Rio de Janeiro.

Métodos. Estudo de coorte retrospectivo baseado em dados de 2.269 casos de tuberculose multirresistente no período 2000-2016. Fatores associados aos desfechos de falha terapêutica, perda de seguimento e óbito em pacientes com resistência primária e adquirida foram analisados em modelos de regressão bivariada e multivariada.

Resultados. Observou-se resistência primária em $14,7 \%$ dos casos de tuberculose multirresistente. Desfechos desfavoráveis ocorreram em 30,3\% no grupo com resistência primária e 46,7\% no grupo com resistência adquirida. Verificaram-se diferenças significativas quanto às características demográficas e clínicas entre os dois grupos. Proporcionalmente, o grupo com resistência primária apresentou mais casos em pacientes do sexo feminino ( $46,4 \%$ vs. $33,5 \%$ no grupo de resistência adquirida), caucasianos $(47,3 \%$ vs. $34 \%$ ) e com escolaridade $\geq 8$ anos (37,7\% vs. $27,4 \%$ ). A tuberculose extensivamente resistente foi associada a uma chance 12,2 vezes maior de falha terapêutica que a tuberculose multirresistente e a chance de presença de comorbidades foi 2 vezes maior no grupo com resistência primária. A chance de ocorrência de tuberculose extensivamente resistente foi 5,43 maior no grupo com tuberculose multirresistente adquirida. Doença bilateral e escolaridade $<8$ anos foram associados à falha terapêutica em ambos os grupos. Estar encarcerado foi associado a uma chance 8 vezes maior de perda de seguimento no grupo com resistência primária. A conversão da cultura após seis meses de tratamento foi um fator de proteção para todos os desfechos.

Conclusões. Os casos de tuberculose multirresistente com resistência primária constituem um reservatório de transmissão distinto que está relacionado a outras doenças crônicas associadas a uma taxa maior de tuberculose. Os resultados ruins observados no Estado do Rio de Janeiro podem contribuir para aumentar a transmissão da tuberculose multirresistente primária, favorecendo a resistência aos medicamentos.

Palavras-chave Tuberculose; monitoramento epidemiológico; resistência a medicamentos; resultado do tratamento; Brasil. 